\title{
Abdominal pain: a synthesis of recommendations for its correct management
}

\author{
Daniela Tirotta, ${ }^{1}$ Annalisa Marchetti, ${ }^{2}$ Mariangela Di Lillo, ${ }^{3}$ Fulvio Pomero, ${ }^{4}$ Roberta Re,${ }^{5}$ Michele Meschi, ${ }^{6}$ \\ Domenico Montemurro, ${ }^{7}$ Paola Gnerre, ${ }^{8}$ Chiara Bozzano, ${ }^{9}$ Micaela La Regina ${ }^{10}$ \\ ${ }^{1}$ Medicina Interna, Ospedale Cervesi, Cattolica (RN); ${ }^{2}$ Medicina d'Urgenza, Ospedali Riuniti, Ancona; ${ }^{3}$ Medicina di Ac- \\ cettazione e di Urgenza, Ospedali Riuniti, Marche Nord, Fano (PU); ${ }^{4}$ Dipartimento di Medicina Interna, \\ Ospedale S. Croce e Carle, Cuneo; ${ }^{5}$ Dipartimento di Medicina Interna, Ospedale Maggiore della Carità, Novara; \\ ${ }^{6}$ Dipartimento di Medicina e Diagnostica, Ospedale Santa Maria, Parma; ${ }^{7}$ Dipartimento di Medicina Interna, Ospedale \\ S. Bortolo, Vicenza; ${ }^{8}$ UOC Medicina Interna, Ospedale San Paolo, Savona; ${ }^{9}$ UO Medicina Interna, Ospedale San Donato, \\ Arezzo; ${ }^{10}$ Medicina Interna, Ospedale Sant'Andrea, La Spezia, Italy
}

\begin{abstract}
Abdominal pain represents one of the most important diagnostic challenges for any physician and its correct interpretation and management require a proper systematic approach and sometimes an urgent action. Moreover the guidelines that can be referred to for indications about the most adequate management procedures are few and often focused only on radiologic management. Consequently, the approach to abdominal pain is often empirical. Therefore, we propose a review of the literature on the diagnosis of abdominal pain, which may contribute to improve the diagnosis and treatment of this complex condition through a systematic review of the evidences available in this field. As to our methodology, we conducted an extensive search in the main guideline databases (SIGN, ICSI, NICE, National Guideline Cleringhouse, CMA Infobase, NZ Guidelines Group, National System Guidelines, Clinical Practice Guidelines Portal, eGuidelines), using as key words abdominal pain and abdominalgia. The guidelines were assessed according to the 2010 Italian version of the AGREE (Appraisal of Guidelines, Research and Evaluation II) methodology. Afterwards we formulated our main recommendations associated with the corresponding levels of evidence and focused our attention on some grey areas, which we investigated with further research using Medline and the main systematic review databases (Cochrane database). The four main grey areas investigated were: hospital admission criteria, prognostic stratification, need for analgesic treatment and possibility of attributing abdominalgia to an abdominal pain syndrome. We then formulated our consesus-based recommendations on the grey areas. Abdominal pain management remains a complex issue for internists. As with other diagnostic challenges, it would be advisable to develop additional guidelines based on a multidisciplinary approach and not only focused on radiological management.
\end{abstract}

Correspondence: Daniela Tirotta, Medicina Interna, Ospedale Cervesi di Cattolica (AUSL Romagna), via Beethoven 1, 47841 Cattolica (RN), Italy.

Tel.: +39.0541966291 - Fax: +39.0541966290.

E-mail: danitirotta@libero.it

Key words: Abdominal pain; management; hospital admission.

Conflict of interest: the authors declare no potential conflict of interest.

See online Appendix for Summary.

Received for publication: 9 May 2014.

Revision received: 16 June 2014.

Accepted for publication: 25 June 2014.

This work is licensed under a Creative Commons Attribution NonCommercial 3.0 License (CC BY-NC 3.0).

(C) Copyright D. Tirotta et al., 2015

Licensee PAGEPress, Italy

Italian Journal of Medicine 2015; 9:193-202

doi:10.4081/itjm.2015.515

\section{Introduction}

Abdominal pain represents one of the most important diagnostic challenges for any physician and its correct interpretation and management require a proper systematic approach and sometimes an urgent action. Moreover the guidelines that can be referred to for indications about the most adequate management procedures are few and often focused only on radiologic management. Therefore, we propose a review of the literature on the diagnosis of abdominal pain, which may contribute to improve the diagnosis and treatment of this complex condition through a systematic review of the evidences available in this field.

\section{Definition of the subject}

Abdominal pain is classified as acute or chronic based on an arbitrary cut-off of 12 weeks. To make a differential diagnosis is very complex and requires an accurate understanding of the medical history and a comprehensive physical examination. Nonetheless the cause remains unknown in $30 \%$ of cases. ${ }^{1,2}$ 


\section{Differential diagnosis of abdominal pain}

The differential dignosis of abdominal pain can be based on physiopathologic categories (Table 1), type of symptoms or site of pain. ${ }^{1}$

\section{Methodology}

\section{Abdominal pain management: collection of evidence}

A search was made in the main databases using $a b$ dominal pain or abdominalgia as keywords:

- Scottish Intercollegiate Guidelines Network (SIGN);

- Institute for Clinical System Improvement (ICSI);

- National Institute for Health and Clinical Excellence (NICE) (NHS evidence);

- National Guideline Cleringhouse;

- Canadian Medical Association, CMA Infobase;

- New Zeland Guidelines Group;

- National Sistem Guidelines;

- Clinical Practice Guidelines Portal;

- eGuidelines.

The guidelines were assessed using the 2010 Italian version of the AGREE (Appraisal of Guidelines, Research and Evaluation II) methodology.

We found and reviewed 7 guidelines (Table 2). ${ }^{11-18}$ Next, we extrapolated the main recommendations associated with the corresponding levels of evidence and we conducted a methodological analysis. ${ }^{11}$

\section{Grading levels of evidence}

We referred to the evidence grading scheme developed within the framework of the Progetto Nazionale Linee Guida (National Project Guideline), available online at $h t t p: / / w w w . p n l g . i t / d o c / m a n u a l e:$ i) level I: evidence from multiple clinical controlled trials and/or systematic reviews of randomized studies; ii) level II: evidence from a single randomized study with an ade- quate design; iii) level III: evidence from non randomized cohort studies or their meta-analysis; iv) level IV: evidence from case-control retrospective studies or their meta-analysis; v) level V: evidence from case series with no control group; vi) level VI: evidence from opinions of experts or expert committees as indicated in the guidelines or consensus conferences.

We then focused our attention on the main 4 grey areas we identified, and which we then investigated with further research including also minor evidence: studies from primary bibliographic databases and other integrative reviews.

In particular, for hospital admission criteria, we used the following research criteria: ("Abdominal Pain"[Mesh]) AND "Hospitalization"[Mesh], Limits Activated: Humans, English, French, Italian, All Adult: $19+$ years, years: 2000.

For prognostic stratification and management, we adopted the following strategy published in Pubmed: ("Abdominal Pain"[Mesh]) AND "Sensitivity and Specificity"[Mesh]) AND "Diagnosis"[Mesh], also ("likelihood ratio"[Mesh]) AND "Sensitivity and Specificity"[Mesh]) and AND "abdominal pain",[Mesh]), Limits Activated: Humans, English, French, Italian, All Adult: $19+$ years, years:2000.

As to the need for analgesic treatment, we relied on the Cochrane Database and Pubmed with mesh analgesia AND abdominal pain.

\section{Summary of abdominal pain guidelines}

To create a more synthetic and systematic organization of recommendations extracted from the guidelines, we have divided them into four subchapters, which correspond to four clinical syndromes: patients with abdominal pain/acute non-specific abdominal pain, patients with abdominal pain in one quadrant, patient with abdominal pain and fever or suspected

Table 2. Evidence table.

\begin{tabular}{|c|c|}
\hline Guidelines & AGREE \\
\hline Cartwright and Knudson, $2008^{11}$ & $(38+75+39) / 3=50.6$ \\
\hline $\mathrm{ACR}, 2008^{12}$ & $(56+67+75) / 3=66$ \\
\hline American College of Emergency Physicians, $2010^{13}$ & $(59+57+56) / 3=57.3$ \\
\hline Society of American Gastrointestinal and Endoscopic Surgeons, $2007^{14}$ & $(83+81+81) / 3=81.6$ \\
\hline $\mathrm{ACR}, 2008^{15}$ & $(57+55+75) / 3=62.3$ \\
\hline ACR, $2010^{16}$ & $(60+60+74) / 3=64.6$ \\
\hline ACR, $2010^{16}$ & $(59+86+75) / 3=73.3$ \\
\hline National Collaborating Centre for Nursing and Supportive Care, $2008^{17}$ & $(130+133+132) / 3=131$ \\
\hline World Gastroenterology Organisation, $2009^{18}$ & $(60+67+74) / 3=67$ \\
\hline
\end{tabular}

AGREE, Appraisal of Guidelines, Research and Evaluation II; ACR, American College of Radiology. 
acute appendicitis and, finally, patient with abdominal pain secondary to suspected irritable bowel syndrome (IBS) and, finally, patients with abdominal pain/acute non specific abdominal pain.

Guidelines for this clinical picture: two guidelines focus on patients with acute abdominal pain/diffuse non specific abdominal pain. ${ }^{14,19}$

Recommended strategy: i) white blood cell count; ii) prescription of abdominal ultrasound (US); iii) prescription of abdominal computed tomography (CT) with contrast agent (CA); iv) prescription of additional imaging exams; v) diagnostic laparoscopy (DL).

Outcome: diagnosis.

Recommendations: i) the white blood cell count confirms, but does not exclude, appendicitis even if it is negative (level VI); ii) DL can be safely performed in selected patients (level III), but it is not indicated before non invasive procedures, despite it can be superior to observation (level III). This procedure can be considered for patients with acute abdominal pain of unknown origin, after an appropriate clinical assessment and imaging exams (VI).

\section{Patients with abdominal pain in one quadrant}

Guidelines for this clinical picture: four guidelines provide recommendations for this symptom. ${ }^{15,16,19}$

Recommended strategy: i) request for amylase and lipase tests; ii) prescription of abdominal US; iii) prescription of abdominal CT with CA; iv) prescription of additional imaging exams.

Outcome: diagnosis.

Recommendations: i) for the preliminary differential diagnosis of epigastric pain, the pancreatic enzyme tests are recommended (level VI); ii) for the preliminary differential diagnosis of pain in the upper right quadrant, abdominal US is recommended (level VI); iii) for the differential diagnosis of pain in the lower quadrants, abdomen-pelvis CT is in general the most accurate method. However for children, US is preferable. Also for pregnant women, US is preferable and, if no diagnosis is obtained, abdominal nuclear magnetic resonance (NMR) imaging should be prescribed (level VI); iv) for the differential diagnoses of pain in the lower left quadrant, abdomen-pelvis CT is appropriate, but it is associated with a high risk of radiation exposure. Other techniques involve an intermediate appropriateness risk with lower radiation doses. During pregnancy, indications are similar, but the most appropriate techniques are US, also trans-rectal and trans-vaginal US, and, to a lesser extent, the barium enema (level VI).

\section{Patient with abdominal pain and fever or suspected acute appendicitis}

Guidelines for this clinical picture: two guidelines provide recommendations for these symptoms. ${ }^{13,16} \mathrm{Six}$ groups of patients are defined: i) adults with suspected acute appendicitis; ii) children with suspected acute appendicitis; iii) patients with acute abdominal pain and fever after surgery; iv) patient with acute abdominal pain and fever with negative CT for abscesses in the previous 7 days; v) patient with acute abdominal pain and fever not operated; vi) pregnant patient.

Recommended strategy: i) use of clinical elements (signs and symptoms); ii) use of abdomen-pelvis CT with intra-venous or oral CA; iii) abdominal US; iv) use of $\mathrm{CT}$ without $\mathrm{CA}$; v) use of alternative techniques (abdominal MR imaging with and without CA, abdominal radiography with gastrografin, gallium and labelled leukocyte scintigraphy).

Outcome: diagnosis.

Recommendations: clinical elements (signs and symptoms) are useful to stratify the patients and make decisions on lab tests, imaging, management (discharge, observation, surgical assessment) (level III). The abdomen-pelvis CT scan confirms or excludes an acute appendicitis and the CA enhances the sensitivity of this tecnique (level III). Abdominal ultrasound confirms, but does not exclude an acute appendicitis and is recommended in children and, as a preliminary examination, in adults (level III). Alternative imaging techniques are less appropriate, but often pose fewer risks associated with radiation exposure.

\section{Patient with abdominal pain and hemodynamic instability or severe abdominal distension and a clear indication for laparotomy}

One guideline provides recommendations for this clinical picture. ${ }^{14}$

Recommended strategy: diagnostic laparotomy.

Recommendation: diagnostic laparotomy should be avoided for the former clinical picture, while it has a limited role for the latter (level VI).

\section{Patient with abdominal pain secondary to suspected irritable bowel syndrome}

Two guidelines provide recommendations for this clinical picture. ${ }^{17,19}$

Recommended strategy: i) examination and confirmation of the IBS; ii) investigation of red flags.

Recommendations: IBS must be suspected in case of: abdominal pain or discomfort, change in bowel habits, bloating for at least 6 months ${ }^{16}$ (level III), abdominal pain/discomfort and chronicity, intermittent pain, previous episodes, relief of pain with defecation, unusual night time diffuse and localized pain ${ }^{18}$ (level VI).

IBS can be confirmed if: abdominal pain/discomfort is relieved by defecation or is associated with altered bowel frequency or altered stool consistency accompanied by at least two of the following symptoms: straining, urgency, incomplete evacuation/abdo- 
minal bloating/worsening by eating/mucus in the stools $^{16}$ (level I-III); on the basis of the Roma III criteria: i) presence of symptoms for at least 6 month before diagnosis; ii) recurrent abdominal pain or $d i-$ scomfort for more than 3 days a month in the last 3 months; iii) at least 2 of the following features: clinical improvement by defecation; association with a change in bowel frequency or stool consistency ${ }^{18}$ (level I-III).

Patients should be examined to identify any red flags: unexplained or unintentional weight loss, rectal bleeding, family history of bowel or ovarian cancer, change in bowel habits to more frequent stools persisting for more than 6 weeks in a patient aged over 60 years; also: anemia, abdominal/rectal masses, inflammatory bowel disease ${ }^{17,18}$ (level I-III).

\section{Main drawbacks of the assessed guidelines}

These guidelines focus primarily on abdominal pain, therefore they exclude other diseases associated with other symptoms (such as a change in bowel habits or melaena/hematemesis). Therefore abdominal pain associated with inflammatory bowel syndrome or anaemia or a change in bowel habits should suggest a gastroenteric disease or an inflammatory bowel disease or, also, a neoplastic etiology based on clinical criteria. However this topic is outside the scope of this monograph.

Abdominal pain classified by quadrants is covered by various guidelines issued by radiological societies and can be a bias in terms of clinical methodology.

\section{Grey areas}

Four unanswered background questions have been identified in the guidelines examined: i) Are there any criteria to decide whether to keep at the hospital or discharge a patient with acute abdominal pain? ii) For the purpose of abdominal pain stratification what is the value of the abdominal pain severity scores? iii) Should the degree of pain be always assessed? Should the analgesic therapy be always deferred initially? iv) Are there any criteria to associate a patient with abdominal pain to a specific abdominal syndrome?

\section{Background question 1}

Are there any criteria to decide whether to keep at the hospital or discharge a patient with acute abdominal pain?

Recommendation: the decision should be substantially based on the clinical picture considering the usual state of health of the patient, the identification of any systemic involvement associated with the main disease and the potential evolution of the category of the suspected disorder (level VI). These variables should lead to a preliminary assessment of the severity and the presence of any potential indications of surgical abdomen (level VI). ${ }^{20-22}$

Despite in some case reports, the rate of inappropriate abdominal emergencies ranges from $5 \%$ to $82 \%$, ${ }^{18}$ at present the flow of patients with abdominal pain is not managed on a codified basis in emergency departments. In the literature some algorhytms are reported that can be applied in hospitals. They are based on emergency level, maximum waiting time before treatment and most appropriate structures to treat these cases.

The primary goal is to define the severity of abdominal pain: i) emergency (abdominal pain with generalized signs of severity, signs of shock). In this case, the team should initiate resuscitation measures and refer the patient to the surgery ward or the intensive care unit; ii) urgency (abdominal pain with localized signs of severity). In this case (evident local signs: occlusive/peritoneal syndrome for suspected appendicitis, cholecystitis, sigmoiditis, strangulated hernia), the team should agree on the necessary tests and examinations to perform and the medical/surgical strategy to implement; iii) relative urgency (no signs of severity). If the case is relatively urgent (abdominal pain without local or generalized signs of severity), the team should assess the following: i) Is the diagnosis confirmed, suspected or unclear? ii) Should the patient be admitted to hospital and why? iii) Which radiographic examinations should be requested and when (are they urgent or can they be deferred)?

The identification of an emergency should be based on: clinical signs (signs of shock: tachycardia, hypotension, bleeding, confusion, respiratory distress, anuria); biological signs (anaemia, dehydration, acidosis, kidney failure). The diagnosis is sometimes evident and requires immediate surgery, whereas on other occasions an urgent abdominal CT scan may be required.

The main causes for an emergency are: i) ruptured ectopic pregnacy or other cause of hemoperitoneum; ii) ruptured aneurysm; iii) occlusion and peritonitis detected late or in fragile patients (children, elderly, immunodepressed); iv) mesenteric ischemia or colic necrosis; v) acute necrotizing-hemorrhagic pancreatitis.

These causes require prompt resuscitation measures and often surgery, which correlates to the state of the patient at time of admission, the decision-making speed and the quality of the resuscitation procedure.

The second goal is to rule out a surgical abdomen, which can be identified by a clinical examination in $70 \%$ of cases. Sometimes the clinical findings are not specific, therefore either the patient is promptly admitted to hospital and kept under observation or an US-CT is performed to find more indications and refer him/her to a medical department or to the surgical ward.

The following should be assessed in particular: worsening of pain on palpation, a defensive reaction (peritoneal irritation), peritonism (sign of abdominal sepsis), 
more peritoneal signs (pain when breathing in and out and coughing), no peristalsis or peristalsis with metallic sounds. These signs are associated with hospital admission in $70 \%$ of the cases, if they are simultaneous.

\section{Background question 2}

For the purpose of abdominal pain stratification what is the value of the abdominal pain severity scores?

Recommendation: scores are helpful to grade the risk, however they cannot lead to a conclusive diagnosis (level I). ${ }^{23-34}$

In a recent health technology assessment ${ }^{36}$ the use of a diagnostic tool seems useful to confirm the diagnosis of acute appendicitis, but not to exclude it.

Among potentially helpful scores, we can name:

- Alvarado score: it can be used to assess pain in the right iliac fossa [a score $\geq 7$ has a positive predictive value (PPV) of $84 \%$ for appendicitis and, in some studies, if $\geq 4$, it suggests admission to hospital]. Furthermore, it can also be used outside hospital settings.

- APACHE score II, Ranson score: scores for acute pancreatitis (Table 3).

Ranson score: early assessment system (mortality of $0-3 \%$ if score $<3,11-15 \%$ if score $\geq 3,40 \%$ if $\geq 6$ ). Although the system is still in use, a recent metaanalysis has shown it has a poor predictive value. ${ }^{22}$

APACHE score II: originally it was used in the Intensive care Unit for critically-ill patients. At present it is also used in acute pancreatitis. It has a good negative predictive value (NPV) and a modest PPV for severe acute pancreatitis. If the score is $<8$, mortality is below $4 \%$; if it is $>8$, mortality is $11-18 \%$. Drawbacks: it is complex and does not differentiate necrotizing and interstitial pancreatitis from sterile and infected necrosis. Furthermore it has a PPV in the first $24 \mathrm{~h}$ [the predictive value can be enhanced using the addition of a body mass index score to APACHE II (known as APACHE O)].

- Ripasa score: it is used when acute appendicitis is suspected. Cut-off is 7.5 , sensitivity (SE) is $88 \%$, specificity (SP) is $67 \%$, PPV is $93 \%$ and NPV is $53 \%$.
Among other relevant factors we can include: i) marked lymphopenia and neutropenia; ${ }^{23,24}$ ii) the Roma III criteria and the Carnet's sign for functional etiology; iii) plasma lactates; iv) pain chronicity seems to be associated with low education, female gender and a history of abuse in young age.

\section{Background question 3}

Should the degree of pain be always assessed? Should the analgesic therapy be always deferred initially?

In case of discharge from hospital, an analgesic treatment can be prescribed and followed up in the short term. In case of admission to hospital, analgesics can be administered, while the patient is waiting in the emergency department (sometimes for hours). Actually both a rehydration therapy (particularly in case of sepsis) and an analgesic therapy are recommended and don not seem to affect the management of the patient (level I). . $^{36-38}$

A recent Cochrane systematic review recommends the use of opioid analgesics to control acute abdominal pain, since they cannot affect the diagnosis, nor the selection of treatment. ${ }^{39}$

\section{Background question 4}

Are there any criteria to associate a patient with abdominal pain to a specific abdominal syndrome?

In the literature, just like in the clinical practice, no structured approach to the differential diagnosis is available. The clinical assessment should lead to the identification of a specific syndrome on the basis of the characterization of pain and the concomitant symptoms (e.g., dull pain indicative of acute mesenteric ischemia, some types of bleedings and chemical peritonitis; progressive pain associated with inflammation, sepsis, distension of solid organs; colic pain usually associated with occlusion of hollow organs), vital signs (the most sensitive are heart rate, body temperature, respiratory rate, blood pressure and urinary

Table 3. Predictive severity scores in pancreatitis.

\begin{tabular}{lccccc}
\hline Time (h) & SE (\%) & SP & PPV & NPV & Accuracy \\
\hline 0 & & & & & 87 \\
Clinical & 44 & 95 & 65 & 89 & 77 \\
Apache II & 63 & 81 & 46 & & 88 \\
\hline 24 & & & & 90 & 87 \\
Clinical & 59 & 96 & 75 & 93 & 89 \\
Apache II $>10$ & 71 & 91 & & 92 & 69 \\
\hline 48 & & & 76 & 91 & 88 \\
Clinical & 66 & 95 & 37 & 93 & \\
Ranson $>2$ & 75 & 98 & 71 & & \\
Apache II $>9$ & 75 & 92 & & & \\
\hline
\end{tabular}

SE, sensitivity; SP, specificity; PPV, positive predictive value; NPV, negative predictive value. 
output), assessment of the clinical picture (such as history of unoperated abdominal aneurysm, use of anticoagulants) (level VI).

Abdominal pain can be included in one of the following clinical syndromes: i) localized or diffuse peritonitic syndrome; ii) unspecific abdominal pain: pain syndrome of non-gastric or medical origin, irritable bowel disease; iii) vascular syndrome; iv) occlusal syndrome. ${ }^{40}$

\section{Peritonitic syndrome}

The diagnosis of peritonitis is generally clinical, although the onset is often insidious. Recommendations are based on consensus (level VI). ${ }^{41,42}$

Symptoms include: fever and shivering; abdominal pain or discomfort ( $70 \%$ of patients); unexplained or rapidly progressive encephalopathy; diarrhea; ascites unresponsive to diuretic therapy; ileus; nausea and anorexia (that can precede pain) and vomiting (secondary to organ pathology, such as peritoneum obstruction or inflammation). The patient appears to be very unwell, temperature is often above $38^{\circ} \mathrm{C}$, although sepsis may also be accompanied by hypotermia. Subsequently the patient becomes oliguric and anuric until shock develops.

Initially pain can be poorly localized (abdominal wall), then it becomes more severe and localized (abdominal wall) and can be exacerbated by coughing, breathing in and out (SE 37\%, SP 94\%, PPV 70\%, NPV 79\%).

Signs and symptoms can be misleading or concealed by: diabetes mellitus or other immunodepressed conditions; advanced age; ascites (cirrhosis or nephrotic syndrome); peritoneal dialysis; steroid treatment.

Accurate management includes differentiation between primary and secondary peritonitis.

Primary peritonitis is diffuse and/or is not associated with a history of gastro-intestinal disease. In other cases (localized or diffuse peritonitis with known gastro-intestinal disease), peritonitis is likely to be secondary.

In case of primary peritonitis, it is recommended to perform a chemical and physical test and a culture of a sample of ascitic tissue or from the peritoneal dialysis and to initiate an antibiotic and support treatment to be continued for 7 days. If the patient does not respond, repeat the culture tests and remove the catheter.

The clinical history includes recent abdominal surgery, previous episodes of peritonitis, travels, immunosuppressive therapy, comorbidity (chronic inflammatory bowel disease, diverticolitis, peptic ulcer), which can predispose to abdominal infections.

\section{Non-specific abdominal pain}

Non-specific abdominal pain is defined as a pain of unknown origin at the time of admission to hospital and requires surgery (in particular the patient has no fever, no tachycardia and no abdominal tenderness). ${ }^{43,44}$ In general this is a self-limiting disorder (in some more favorable cases the patient has no symptoms at 5 years in $77 \%$ of cases), which, however, leads to $13-40 \%$ of admissions for surgery. In this case, observation can be important. The patient should be re-assessed within $24 \mathrm{~h}$, because this might be an unusual presentation of other acute diseases, such as acute miocardial infarction and ischemic colitis.

Recent cases from etiological studies have shown that $10 \%$ of patients have a neoplastic disease (in particular above 50 years of age). Sometimes it is caused by gynecologic and urologic diseases, functional diseases, such as the irritable bowel syndrome (see guidelines previously examined); sometimes it is caused by coeliac disease. Two disorders are often reported: mild alcoholic pancreatitis, crisis secondary to sicklecell anemia.

Furthermore, a drug-related etiology should also be considered: non-steroidal anti-inflammatory drugs, erythromycin, other antibiotics (colitis due to clindamycin, cephalosporins, ampicillin, amoxicillin) and sub-occlusion secondary to constipation.

\section{Vascular abdominal syndrome}

Vascular abdominal syndromes represent a severe abdominal emergency, are typical of advanced age and, although rare ( $1 \%$ of causes for acute abdominal pain), they are associated with a high mortality rate (10-90\% of cases). ${ }^{45-49}$

The clinical picture in the early stages is unspecific and similar to other abdominal syndromes (peritonitis, occlusion). Pain can be acute, sudden (arterial embolism or thrombosis) or slowly progressing (ischemia by non occlusal causes or venous mesenteric thrombosis), associated with nausea, vomiting and change in bowel habits.

Initially the physical examination is unspecific, whereas at later stages ( $>6 \mathrm{~h}$, necrotic evolution), the patient develops typical signs of peritonitis, hemodynamic instability and signs and symptoms of sepsis with failure of multiple organs.

For an accurate and early diagnosis it is fundamental to identify the main risk factors when the medical history is assessed.

Various pathogenic entities can be recognized with the following characteristics: myocardial infarction/intestinal ischemia; aortic aneurysm rupturing or fissure, abdominal aneurysm rupturing; abdominal blood vessel thrombosis.

The main consensus-based recommendations for a diagnosis of acute intestinal ischemia (level VI) include: i) significant risk factors: age $>60$ years; atrial fibrillation or history of paroxymal atrial fibrillation, recent myocardial infarction, heart failure, shock, previous arterial embolism; history of abdominal pain 
after eating and weight loss; abdominal pain inconsistent with physical examination findings; nausea, vomiting; ii) suggestive test results, if associated with significant risk factors: leukocytosis, increase of lactate dehydrogenase and creatine phosphokinase, metabolic acidosis.

In case of significant risk factors and hemodyna- mic stability, angiography is recommeded with a simultaneous vasodilator infusion or papaverine infusion in the superiror mesenteric artery, if readily available; otherwise CT angiography is recommended.

If signs of peritonism and hemodynamic instability are present, emergency exploratory laparotomy is recommended with resection of the necrotic intestinal

\section{EMERGENCY}

Clinical signs of shock: confusion, respiratory distress, hemorrhage, anuria, tachycardia, hypotension

Biological signs: severe anemia, dehydration, acidosis, renal failure
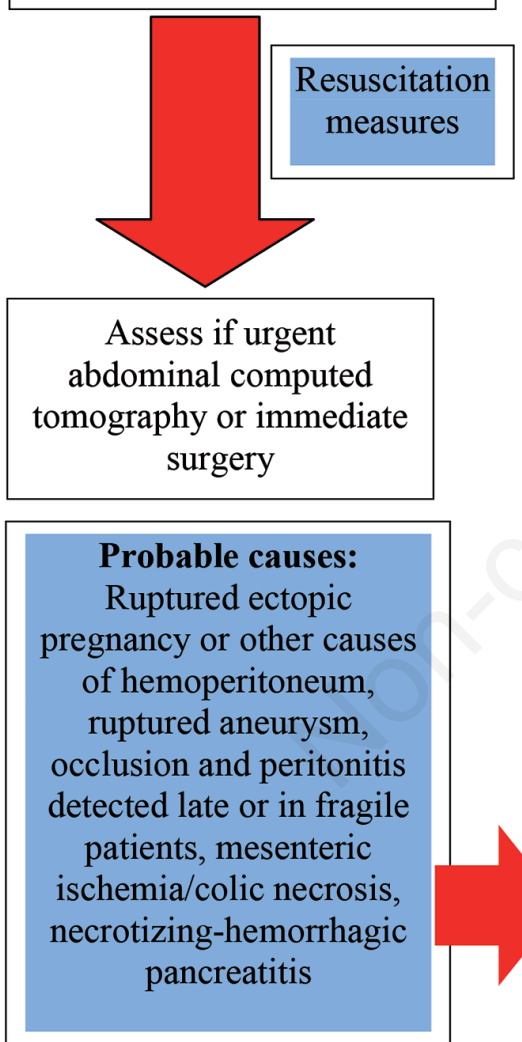
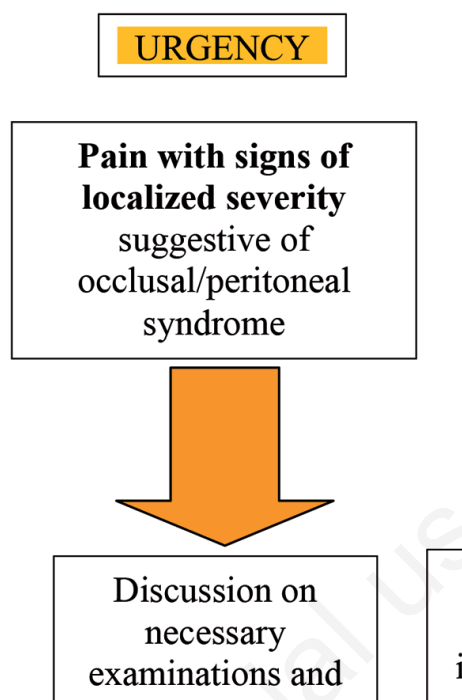
and/or surgery

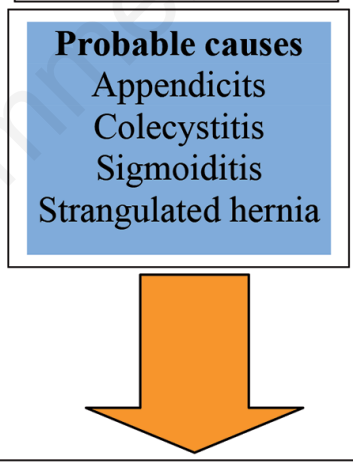

i) Is diagnosis confirmed, suspected or indefinite?

ii) Should the patient be admitted to hospital and why? iii) Which radiographic examinations are required?

Urgent or deferred request?

\section{pain with no signs of severity}
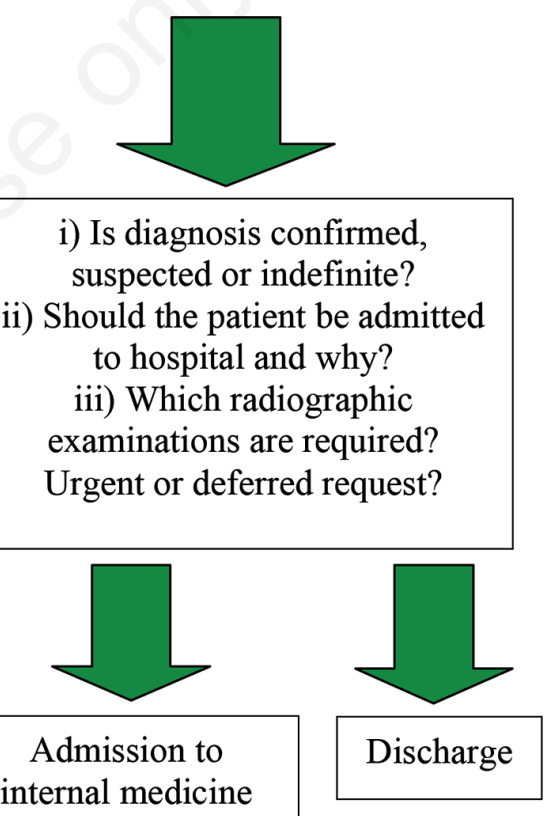
ward

\section{$\sqrt{\text { Assess if signs of surgical }}$ abdomen: \\ i) Tenderness upon palpation} ii) Defence

iii) Signs of peritonism

iv) Pain when breathing in and out and coughing

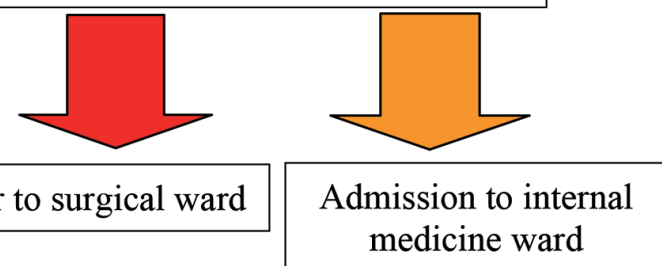

Figure 1. Abdominal pain: diagnostic flow-chart. 
tract and revascularization (embolectomy, thrombectomy and intra-arterial infusion of papaverine and vasodilators).

The following clinical factors are suggestive of aortic aneurysm fissure or rupturing (the main prognostic factor is the size of the aneurysm): abdominal and lumbar pain, shock, pulsating abdominal mass, synchronous or asynchronous femoral pulses, risk factors (smoking, family history, age $>70$ years, history of atherosclerosis, female sex, diabetes, African race).

Among the diagnostic factors suggestive of a thrombosis of abdominal blood vessels, we highlight (level VI): lower limb ischemia (no pulse, pallor, paresthesia, pain), signs of mesenteric ischemia, signs of spinal cord infarction.

The preliminary management of a patient with suspected aortic thrombosis requires an immediate $\mathrm{CT}$ angiography of the abdomen. In case of acute aortic thrombosis, emergency laparotomy is required with trombectomy or embolectomy or coronary artery bypass graft. In case of chronic or sub-acute occlusion, the need for emergency surgery depends on the severity of the clinical picture and the occlusion progression. The strategy required most often in these cases is observation and anti-aggregating or anti-coagulating therapy.

\section{Occlusal abdominal syndrome}

The suspicion of an occlusal abdominal syndrome is based on the clinical history of the patient and a number of symptoms: abdominal pain, which is initially cramp-like and then becomes continuous; meteorism, tympanic abdomen, peristalsis with metallic sounds, which, on the contrary are absent in paralytic ileus. Vomiting is frequent in particular in upper occlusions (small intestine); in distal occlusions, fecaloid vomiting can be present.

The main recommendations are based on consensus (level VI). ${ }^{50,51}$

Suggestive clinical history: previous abdominal surgery, radiation therapy, bowel inflammatory disorders, weight loss, constipation, pain in the upper abdominal quadrant (biliary ileus).

An accurate preliminary management of abdominal occlusal syndrome includes a differential diagnosis between a mechanical obstruction and a paralytic ileus.

In case of mechanical ileus of unknown origin, an abdominal CT scan with intravenous CA must be performed immediately. In case of complications, such as strangulation and intestinal ischemia, the patient must be referred to the surgeon for emergency surgery. If no indication for emergency surgery is present, a conservative strategy must be implemented with support therapy (fluids), intestinal decompression (insertion of naso-gastric tube and/or rectal tube) and subsequent assessment; in case this clinical picture persists, an exploratory laparotomy can be useful.
In case of a likely occlusion of the small intestine, oral administration of CA (gastrografin) is required; after $8 \mathrm{~h}$ bowel transit must be assessed in the colon by abdominal radiography. If bowel transit is positive, the patient must be kept under observation for 4-5 days. If no bowel transit is present, an exploratory laparotomy is indicated.

A summary of the management procedure proposed is reported in Figure 1, whereas online Appendix summarizes the proposed management procedure for abdominal pain and the differential diagnosis factors.

\section{References}

1. Tintinalli JE, Gabor DK, Staphczynski S, eds. Tintinalli's emergency medicine: a comprehensive study guide, 6th ed. New York: McGraw-Hill; 2004.

2. Kasper DL, Braunwald E, Fauci AS, et al. Harrison Principi di medicina interna, 16th ed. Milano-New York: Mac Graw-Hill; 2005.

3. Lyon C, Clark DC. Diagnosis of acute abdominal pain in older patients. Am Fam Phys 2006;74:1537-44.

4. Tintinalli JE. Emergency medicine ACEP. Ann Emerg Med 2000;36:406.

5. BMJ Evidence Center. Assessment of chronic abdominal pain. Clinical evidence. Available from: http://bestpractice.bmj.com/best-practice/monograph/767.html Accessed: August 29, 2012.

6. Liu JLY, Wyatt JC, Deeks JJ, et al. Systematic reviews of clinical decision tools for acute abdominal pain. Health Techn Assess 2006;10:47.

7. Meisel JL. Diagnostic approach to abdominal pain in adults. UptoDate 2011. Available from: http://www.uptodate.com

8. Aryal K, Bhowmick A, Beveridge AJ, et al. Hotel NHS and the acute abdomen - admit first, investigate later. Int J Clin Pract 2009;63:1805-7.

9. Penner RM, Majumdar SR. Diagnostic approach to abdominal pain in adult. UpToDate 2011. Available from: http://www.uptodate.com

10. Tintinalli JE, Gabor DK, Staphczynski S, eds. Tintinalli's emergency medicine: a comprehensive study guide, 7th ed. New York: McGraw-Hill; 2010.

11. Cartwright SL, Knudson MP. Evaluation of acute abdominal pain in adults. Am Fam Physician 2008;77:971-8.

12. American College of Radiology (ACR). Appropriateness criteria for acute abdominal pain and fever or suspected abdominal abscess; 2008. Available from: http://www.guideline.gov/content.aspx?id=37926

13. American College of Emergency Physicians. Clinical policy: critical issues in the evaluation and management of emergency department patients with suspected appendicitis. Ann Emerg Med 2010;55:71-116.

14. Society of American Gastrointestinal and Endoscopic Surgeons (SAGES). Diagnostic laparoscopy for acute abdominal pain; 2007. Available from: http://www.guidelines.gov

15. American College of Radiology (ACR). ACR Appropriateness Criteria ${ }^{\circledR}$ left lower quadrant pain; 2008. Available from: http://www.guidelines.gov 
16. American College of Radiology (ACR). ACR Appropriateness Criteria ${ }^{\circledR}$ right upper quadrant pain; 2010. Available from: http://www.guidelines.gov

17. National Collaborating Centre for Nursing and Supportive Care. Irritable bowel syndrome in adults. Diagnosis and management of irritable bowel syndrome in primary care. London (UK): National Institute for Health and Clinical Excellence (NICE); 2008 Feb. 27. Available from: http://www.nice.org.uk

18. World Gastroenterology Organisation (WGO). World Gastroenterology Organisation Global Guideline: irritable bowel syndrome: a global perspective. Munich: World Gastroenterology Organisation (WGO); 2009. p 20.

19. Tirotta D, Marchetti A, Di Lillo M. Il dolore addominale. Quaderni Ital J Med 2013;1:45-68.

20. Schreyer N, Yersin B. L'orientation du patient souffrant d'un syndrome douloureux addominal aigu à domicile. Revue Méd Suisse 2006;2:1844-8.

21. Laméris W, van Randen A, van Es HW, et al. Imaging strategies for detection of urgent conditions in patients with acute abdominal pain: diagnostic accuracy study. BMJ 2009;339:b243.

22. Chiche L, Roupie E, Delassus R, et al. Prise en charge des douleurs abdominales de l'adultes aux Urgences. J Chir 2006; 1:143.

23. De Bernardinis M, Violi V, Roncoroni, L, et al. Discriminant power and information content of Ranson's prognostic signs in acute pancreatitis: a meta-analytic study. Crit Care Med 1999;27:2272.

24. Vege SS. Predicting the severity of acute pancreatitis. UpToDate 2012. Available from: http://www.uptodate.com

25. Deibener-Kaminsky J, Lesesve JF, Kaminsky P. Leukocyte differential for acute abdominal pain in adults. Lab Hematol 2011;17:1-5.

26. Vege SS. Predicting the severity of acute pancreatitis. UptoDate 2011, rev. 2013. Available from: http://www. uptodate.com

27. Swailes E, Rich E, Lock K, Cicotte C. From triage to treatment of severe abdominal pain in the emergency department: evaluating the implementation of the emergency severity index. J Emerg Nurs 2009;35:485-9.

28. Ragsdale L, Southerland L. Acute abdominal pain in older adult. Emerg Med Clin N Am 2011;29:429-48.

29. Takada T, Ikusaka M, Ohira Y, et al. Diagnostic usefulness of Carnett's test in psychogenic abdominal pain. Intern Med 2011;50:213-7.

30. Abid S, Siddiqui S, Jafri W. Discriminant value of Rome III questionnaire in dyspeptic patients. Saudi J Gastroenterol 2011;17:129-33.

31. Chong CF, Adi MI, Thien A. Development of the RIPASA score: a new appendicitis scoring system for the diagnosis of acute appendicitis. Singapore Med J 2010; 51:220-5.

32. Weijenborg PT, Gardien K, Toorenvliet BR. Acute abdominal pain in women at an emergency department: predictors of chronicity. Eur J Pain 2010;14:183-8.
33. Chan MY, Tan C, Chiu MT, Ng YY. Alvarado score: an admission criterion in patients with right iliac fossa pain. Surgeon 2003;1:39-41.

34. Filiz AI, Aladag H, Akin ML, Sucullu I. The role of dlactate in differential diagnosis of acute appendicitis. J Invest Surg 2010;23:218-23.

35. Liu JLY, Wyatt JC, Deeks JJ, et al. Systematic reviews of clinical decision tools for acute abdominal pain. Health Techn Assess 2006;10:47.

36. Geiderman JM, Silka PA. Analgesia in patients with acute abdomen. West J Med 2000;173:209-10.

37. Shabbir J, Ridgway PF, Lynch K, et al. Administration of analgesia for acute abdominal pain suffers in the accident and emergency setting. Eur J Emerg Med 2004; 11:309-12.

38. Rupp T, Delaney KA. Inadequate analgesia in emergency medicine. Ann Emerg Med 2004;43:494-503.

39. Manterola C, Vial M, Moraga J, Astudillo P. Analgesia in patients with acute abdominal pain. Cochrane Database Syst Rev 2011;(1):CD005660.

40. Dang C, Aguilera P, Dang A, Salem L. Acute abdominal pain. Four classifications can guide assessment and management. Geriatrics 2002;57:30-2, 35-6, 41-2.

41. Daley BD. Peritonitis and abdominal sepsis workup. Medscape. Updated: March 29, 2011. Available from: http://emedicine.medscape.com/article/180234-workup

42. Taylor S, Watt M. Emergency department assessment of abdominal pain: clinical indicator tests for detecting peritonism. Eur J Emerg Med 2005;12:275-7.

43. Sanders DS, Azmy IAF, Hurlstone DP. A new insight into non-specific abdominal pain. Ann R Coll Surg Engl 2006;88:92-4.

44. Domínguez LC, Sanabria A, Vega V, et al. Early laparoscopy for the evaluation of nonspecific abdominal pain: a critical appraisal of the evidence. Surg Endosc 2011;25:10-8.

45. American College of Radiology (ACR). ACR Appropriateness Criteria $\AA$ radiologic management of mesenteric ischemia; 2011.

46. Renner P, Kienle K, Dahlke MH, et al. Intestinal ischemia: current treatment concepts. Langenbeck Arch Surg 2011;396:3-11.

47. Oldenburg WA, Lau LL, Rodenberg TJ. Acute mesenteric ischemia. Arch intern Med 2004;164:1054-62.

48. American College of Radiology (ACR). ACR Appropriateness Criteria ${ }^{\circledR}$ abdominal aortic aneurysm: interventional and planning follow-up; 2010. Available from: http://www.guideline.gov/content.aspx?id=32615

49. Lewiss RE, Egan DJ, Shreves A. Vascular abdominal emergencies. Emerg Med Clin N Am 2011;29:253-72.

50. Zielinski MD, Bannon PM. Current management of small bowel obstruction. Adv Surg 2011;45:1-29.

51. American College of Radiology (ACR). ACR Appropriateness Criteria ${ }^{\circledR}$ suspected small-bowel obstruction; 2010. Available from: http://www.acr.org/ /media/ 832F100277004BC69A8C818C7C9BFF33.pdf 